\title{
Polylactide-Based Biodegradable Seaffolds Fabricated by Two-Photon Polymerization for Neurotransplantation
}

\author{
DOI: $10.17691 / \mathrm{stm} 2016.8 .4 .03$
}

Received March 02, 2016

A.V. Koroleva, PhD, Researcher, Nanotechnology Unit';

D.S. Guseva, DSc, Researcher, Department of Neurophysiology2;

N.A. Konovalov, MD, DSc, Professor, Head of the Spinal Neurosurgery Unit3;

T.M. Zharikova, Researcher, Modern Biomaterial Unit';

E.G. Ponimaskin, DSc, Professor, Head of the Department of Cellular Neurophysiology2;

B.N. Chichkov, DSc, Professor, Head of the Nanotechnology Unit';

V.N. Bagratashvili, DSc, Professor, Head of the Unit of Laser Atomic and Molecular Nanotechnologies:;

P.S. Timashev, DSc, Head of the Novel Biomaterial Unit'; Senior Researcher, Laboratory of Laser Chemestry ${ }^{5}$

${ }^{1}$ Laser Zentrum Hannover, 8 Hollerithallee, 30419, Hannover, Germany;

${ }^{2}$ Hannover Medical School, 1 Carl-Neuberg St., 30625, Hannover, Germany;

${ }^{3}$ N.N. Burdenko Research Institute of Neurosurgery, 16, $4^{\text {th }}$ Tverskaya-Yamskaya St., Moscow, 125047,

Russian Federation;

${ }^{4}$ Institute of Regenerative Medicine, I.M. Sechenov First Moscow State Medical University,

8/2 Trubetskaya St., Moscow, 119991, Russian Federation;

5Institute of Photon Technologies, Federal Research Center "Crystallography and Photonics"

of the Russian Academy of Sciences, 2 Pionerskaya St., Moscow, Troitsk, 142190, Russian Federation

The aim of the investigation was to form biodegradable 3D microstructured scaffolds compatible with progenitor neuronal cells as a perspective approach to neurotransplantation.

Materials and Methods. Photosensitive compositions were obtained from branched polylactides using Michler's ketone photoinitiator. The 3D microstructured scaffolds were fabricated by two-photon polymerization method.

Results. 3D microstructured scaffolds with a high spatial resolution have been fabricated from reactive polylactides by two-photon polymerization. The surface of scaffolds represents a structure with a high degree of roughness ( $1 \mu \mathrm{m}$ average roughness). Dissociated hippocampal cells (from embryonic mice) adhere well to the matrix material, and active growth of axons and dendrites takes place. By day 10 of in vitro cultivation a well-branched neuron-glial network is visualized on a 3D construct. The developed structures have been found to possess a high biocompatibility with the primary hippocampal cultures, which suggests their application as matrix-carriers for the cells of the nervous system.

Key words: two-photon polymerization; polylactides; three-dimensional scaffolds; biocompatible materials; neuronal cells.

One of the strategic directions of modern materials science is the application of materials and structures on the basis of biostable and bioresorbable polymers with a desired architectonics [1, 2]. The range of such structure applications is extremely wide: from endoprosthesis replacement (implantology), where articular inserts for artificial joints are made of high-molecular-weight biostable polymers, to regenerative medicine and tissue engineering, when 3D matrices based on bioresorbable polymers are used for restoration of hard tissues [3]. These structures should meet special requirements regarding not only the composition, biological activity, presence of toxic compound traces, but also architectonics, which must mimic replaced tissues or organs, i.e. have specified porosity of the structure and mechanical strength [4].
The method of two-photon polymerization (2PP) allows fabricating 3D micro objects with a high spatial resolution by effective control of microarchitecture during fabrication. These features, along with compatibility with biomaterials, make 2PP method most perspective for solving the problems of creating matrices of the required form and inner architecture for the needs of regenerative medicine $[5,6]$. Complex, porous 3D scaffolds produced by 2PP are especially important for cell culturing in terms of transport of the nutrients within cultured construct [7].

No less important aspect in fabricating 3D matrixes for regenerative medicine is creating of scaffolds based on biodegradable materials. Degradation of such scaffolds during the culture in vitro or in vivo, and regulation of the rates of new tissue formation relative to the rate of synthetic implant bioresorption is another important topic

For contacts: Peter S. Timashev, e-mail: timashev.peter@gmail.com 
of implant research [8]. The possibility of gradual material degradation in the process of the scaffold use in vitro allows the process of tissue growth (regeneration) to run [9], whereas in case of the application of biologically stable materials, it may remain in the organism and initiate inflammatory reactions $[10,11]$. It has been previously shown by us, that 2PP method can be used to fabricate hybrid [12] and hydrogel [13] structures, on which dissociated neuronal cells, obtained from mouse hippocampus, grow. Moreover, these cells were able to form a three-dimensional neural network.

The aim of the investigation was to work out approaches to fabricating branched polylactide-based biocompatible three-dimensional constructs, and to assess the possibility of their application for adhesion of progenitor neuronal cells and further development of neurotransplantats on their basis.

\section{Materials and Methods}

Synthesis of reactive polylactide derivatives. Twostaged synthetic strategy of fabricating tetrafunctional polylactides has been presented in the work [9]. A derivative with $760 \mathrm{Da}$ molecular mass of one arm (or 10.5 lactide links) was chosen for fabricating 3D structures. Chemical composition and molecular mass distribution of the polymer was confirmed by nuclear magnetic resonance (NMR), infrared spectroscopy and gel-penetrating chromatography methods.

Preparing a photosensitive composition for twophoton polymerization. $20 \%$ solution of polymer in dichloromethane (99\%, Sigma-Aldrich, Germany) was prepared to obtain a photosensitive composition. A photoinitiator, Michler's ketone, $1 \%$ by mass, (BASF Kaisten AG, Germany) was introduced in this mixture. The system was agitated at room temperature for $2 \mathrm{~h}$ to obtain a homogenous solution.

Fabrication of three-dimensional structures. The composition was applied on the surface of $12 \mathrm{~mm}$ diameter coverslips (1.13 $\mathrm{cm}^{2}$ sample surface area). Before the application of the composition, the coverslips were placed in $5 \%$ aqueous solution of hydrochloric acid for $6 \mathrm{~h}$, then washed by distilled water for $20 \mathrm{~min}$ in the ultrasound bath. The 3D model, parameters of photoinduced cross-linking and the operating characteristics of a laser system were chosen in accordance with the work [12]. The fabricated structures were washed in $100 \mathrm{ml}$ dichloromethane for $3 \mathrm{~h}$ to remove the unreacted material. The structures then were stored in desiccator at $+2^{\circ} \mathrm{C}$.

A cellular culture of dissociated neurons from mouse hippocampus. Hippocampi were isolated from mouse embryos (on embryonal day 18, E18). The mice (C57BL6J) were withdrawn from the experiment by dislocation of the cervical vertebra according to the protocol for the care and use of the laboratory animals, approved by the Federal Ministry of Health and the Bioethics Committee of the Hannover Medical School (Germany). Dissection of the hippocampus was performed in sterile conditions in HBSS buffer
(Invitrogen, Russia, 24020-091). After fermentative cleavage for $25 \mathrm{~min}$ in $0.25 \%$ trypsin (Invitrogen, Russia, $25200-056)$ at $37^{\circ} \mathrm{C}$, the hippocampi were dissociated by a pipette. After suspension centrifuging at $1,500 \mathrm{~g}$ for $2 \mathrm{~min}$, the cells were resuspended in Neurobasal-A medium (Invitrogen, Russia, 21103-049) enriched with 2\% Supplement B-27 (Invitrogen, Russia, 17504-044), $0.05 \mathrm{mM}$ L-glutamine (Invitrogen, Russia, 25030-024) and $5 \%$ fetal calf serum (NBA medium) (PanEco, Russia, K055). The dissociated cells were inoculated on the structures in $40 \mu \mathrm{l}$ buffer with 9,000 cells $/ \mathrm{mm}^{2}$ initial density. The control group consisted of hippocampal cultures seeded on the coverslips $(18 \mathrm{~mm})$ pretreated with polyethyleneimine (Sigma-Aldrich, Germany, P3143) according to the previously established protocol [14]. Once the cells attached to the structure surfaces or the glass $(2 \mathrm{~h})$, the culture dishes were filled with $1 \mathrm{ml}$ NBA medium.

The medium was changed every 2 days, and only half the volume was replaced in order to preserve trophic factors synthesized by the cultured neurons. The cells were cultivated for 14 days under constant conditions humidified atmosphere containing 95\% air and $5 \% \mathrm{CO}_{2}$.

The cultures were controlled by means of phasecontrast imaging every day for state recording using inverted DMIL HC microscope (Leica, Germany).

Immunocytochemical analysis of the neuronal culture. Immunocytochemical examination of the cells cultured on the scaffolds was performed using a two-stage method. The structures with the cells were fixed in $4 \%$ paraformaldehyde for $30 \mathrm{~min}$ at room temperature. Thereafter the cells were permeabilized with a methanol precooled to $-20^{\circ} \mathrm{C}$ during $3 \mathrm{~min}$. To block nonspecific binding of antibodies, the samples were treated with $2 \%$ calf serum albumin at $37^{\circ} \mathrm{C}$ for 30 min. Incubation of the samples with primary antibodies diluted in $2 \%$ calf serum albumin in PBS was carried out overnight at $4^{\circ} \mathrm{C}$. 1:1,000 BIII-tubulin; mouse monoclonal IgG2a antibodies, 2G10 clone (Sigma-Aldrich, Germany) or 1:50 synaptophysin; rabbit polyclonal $\mathrm{H}-93$ antibodies (Santa Cruz, Germany) were chosen as markers. After several washing stages, the samples were incubated with secondary antibodies conjugated with horseradish peroxidase of goat antibodies against rabbit and mouse $\lg \mathrm{G}(\mathrm{H}+\mathrm{L})(1: 100$; Dianova $\mathrm{GmbH}$, Germany) for $1 \mathrm{~h}$ at room temperature, visualized by incubation with 3-amino9-ethylcarbazole substrate in the buffer of sodium acetate (0.1 mol/L, $\mathrm{pH} 5.2$ ) containing hydrogen peroxide. The structures with the cells were analyzed using Axilolmager microscope (Carl Zeiss, Germany) equipped by AxioCam ICC1 camera and AxioVision 4.8 software.

Scanning electron microscopy. On day 12 of neuronal cell culturing the tissue-engineered constructs were washed twice in PBS solution and fixed with $2.5 \%$ glutaraldehyde for $30 \mathrm{~min}$, and thereafter additionally fixed in $2 \%$ osmium (VIII) oxide for 30 min. After further intensive washing, the samples were dehydrated in the series of ethanol with increasing concentrations (from 
30 to $100 \%$ ) and dried using hexamethyldisilazane. The prepared structures were coated with $5 \mathrm{~nm}$ gold layer. The samples were examined using scanning electron Quanta 400F microscope (FEI Company, USA), equipped with energy dispersive spectroscopy system (EDAX EDS System and Genesis Software, EDAX Inc., USA).

Atomic-force microscopy. For visualization of the matrix-carrier sample surface a scanning probe microscope MultiMode-8 (Bruker, USA) was used. It is provided with PeakForce QNM software (Bruker, USA) capable of mapping the distribution of mechanical characteristics and adhesive forces. To capture the images of the examined object surfaces, the microscope is equipped with silicon RTESP cantilevers (with 105$135 \mu \mathrm{m}$ beam length, 20-80 N/m spring constant, 300$360 \mathrm{kHz}$ resonance frequency, and $<5 \mathrm{~nm}$ radius of tip curvature) without a reflection coating (Veeco, USA). The surface areas sized $5 \times 5$ and $10 \times 10 \mu \mathrm{m}(512 \times 512$ points $)$ were scanned.

Data processing. The results of cell testing and swelling were processed using Origin program. Mean square deviation from the mean was considered an error, differences according to Mann-Whitney U-test were considered to be significant data at $p<0.05$.

\section{Results and Discussion}

Synthesis of reactive polymers and their structuring by two-photon polymerization method. The developed strategy of the synthesis of four functional polylactides allowed us to perform the reaction in two stages and to achieve a high percent of the target product yield $(>90 \%)$ and narrow molecular mass distribution $\left(\mathrm{M}_{\mathrm{w}} / \mathrm{M}_{\mathrm{n}}<1.2\right)$ [9]. The structure and purity of the synthesized polymer were verified by NMR spectroscopy. Earlier, similar synthetic strategies have already been proposed [15], but we optimized the synthesis procedure by means of full polymer conversion running for $15 \mathrm{~min}$ and further polymerization and modification of the sub-product, carried out according to the "single pot" technique without additional stages of separation and purification of the sub-products. The method of 2PP chosen by us enabled fabrication of 3D porous hexagonal structures.

The advantage of the chosen method is in the fabrication of highly specific structures with less than $1 \mu \mathrm{m}$ spatial resolution. Besides, owing to the absence of additional heating of the primary polymer during the initiation of the spatial cross-linking and the accuracy of femtosecond laser in the process of 2PP, the effects of spontaneous cross-linking near the area of laser focusing are not observed, which allows one to reproduce the desired computer model with great precision. The application of reactive 4-arm polylactide provided a high homogeneity of the spatial cross-linking and reduced the effects of material shrinkage after the process of structuring.

Micrographs of 2PP scaffolds, obtained by scanning electron microscopy, visualize the material structure and specified computer model (Figure 1 (a), (b)). Fabricated and washed structures consisted of two layers of hollow cylinders $(160 \mu \mathrm{m}$ high, $100 \mu \mathrm{m}$ inner diameter, $160 \mu \mathrm{m}$ outer diameter) and represented a 3D hexagon. Displacement of the upper layer by $80 \mu \mathrm{m}$ relative to the lower one enables one to form a porous structure, which provides an effective transport of the medium nutritious substances to the cultured cells. Besides, the formed inner projections serve as a supplementary support for cell development and generation of cell outgrowths. Atomic-force microscopy showed, that a great number of irregularities were present on the matrix surface at the submicron level, an average roughness being within $1 \mu \mathrm{m}$ (Figure 1 (c)). Distribution of Young modulus values across the sample surface was obtained by PeakForce QNM method (Figure 1 (d)). It has been estimated that the 2PP method allows forming polymer structures with uniform mechanical properties, the mean value of the surface elastic modulus amounted to $600 \mathrm{MPa}$.

Tissue-engineered polylactide-based constructs compatible with dissociated hippocampal cells. The morphological analysis of dissociated hippocampal cells on day 14 of culturing showed that the growth of cultures on polylactide structures complied with the main developmental signs characteristic to the normal formation of neural networks in the primary culture. On day 1 after seeding the viable cells were attached to the polylactide structures. Generation of neural networks was confirmed by visualization of neuronal cells with antibodies against Blll-tubulin (neuronal marker) and synaptophysin (presynaptic marker), indicating to the development of differentiated neurons capable of generating synaptic contacts. The cells cultured on polylactide structures during 14 days (Figure 2) had both specific markers. This result was comparable with the cell behavior in the control 2D culture on the coverslips.

Electron microscopic analysis of hippocampal dissociated culture, grown on the polylactide structures also showed that morphologic composition of the nervous cells corresponds to the neurons grown on the control coverslips coated with polyethyleneimine. A well-developed dendrite tree with a typical morphology of dendritic spines was observed on the scaffold ring surfaces and in the gaps between the solitary structure segments, proving that the given architecture of the tissueengineered construct promoted the neuron growth and synapse formation in the culture of 3D cells (Figure 3 ).

Conclusion. A synthetic strategy of fabricating reactive lactide derivatives possessing no toxic properties has been worked out. Scaffold structures with high spatial resolution have been formed from this by 2PP method. The initial cells of the hippocampal dissociated culture were found to interact with the matrix-carrier surface, and thereafter an active growth of axons and dendrites took place. Immunohistochemical analysis with neuronal marker $\beta$ III-tubulin and postsynaptic marker synaptophysin testifies to the formation of the functional neuronal network in 3D scaffold. 


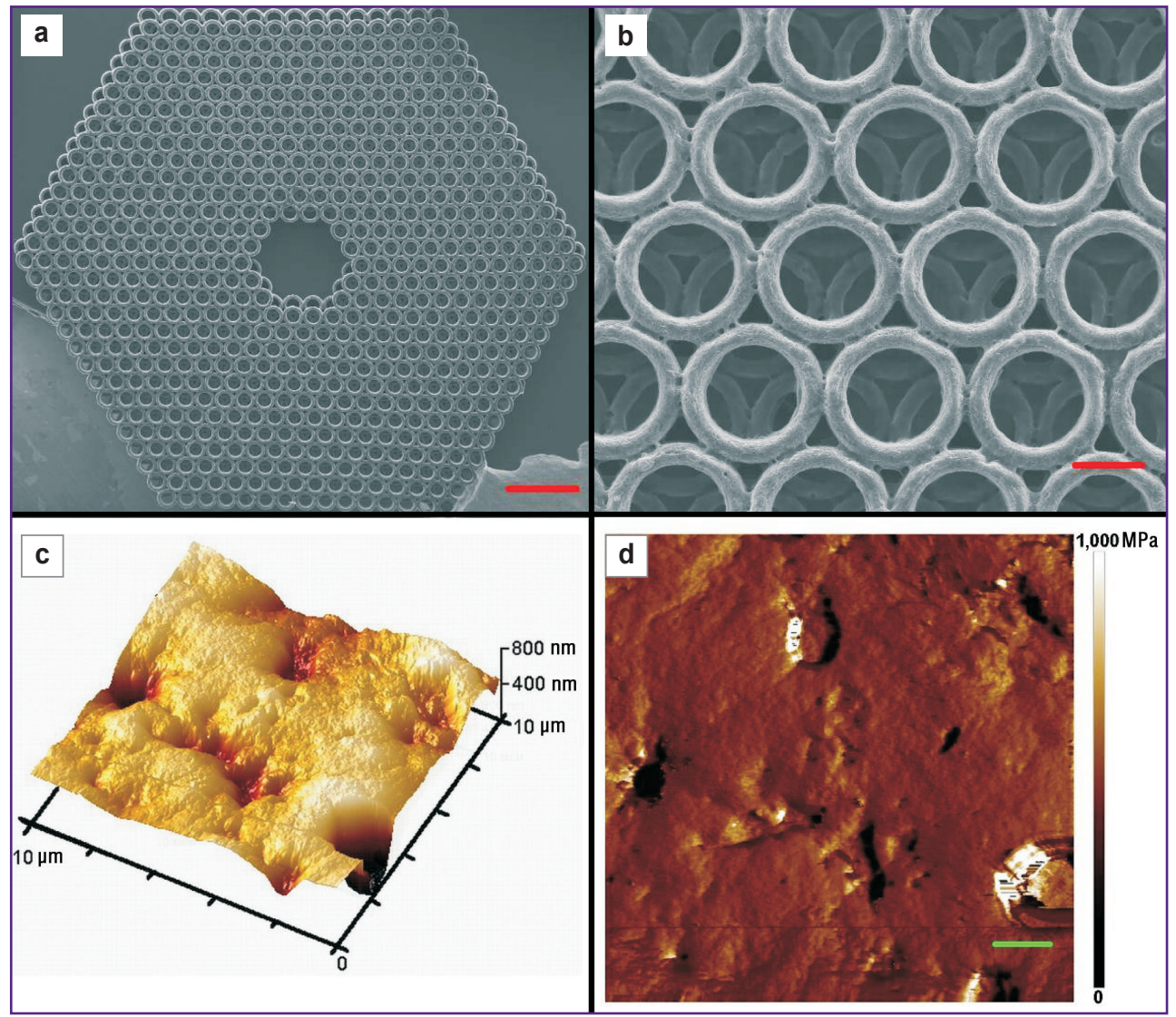

Figure 1. Scanning electron microscopy. Micrographs of the fabricated structures; scale bar $1 \mathrm{~mm}(\mathrm{a})$ and $100 \mu \mathrm{m}$ (b). Atomic-force microscopy. The image (field $10 \mu \mathrm{m}$ ), obtained in the mode of measuring the hight (c) and Young's modulus (d); scale bar $1 \mu \mathrm{m}$
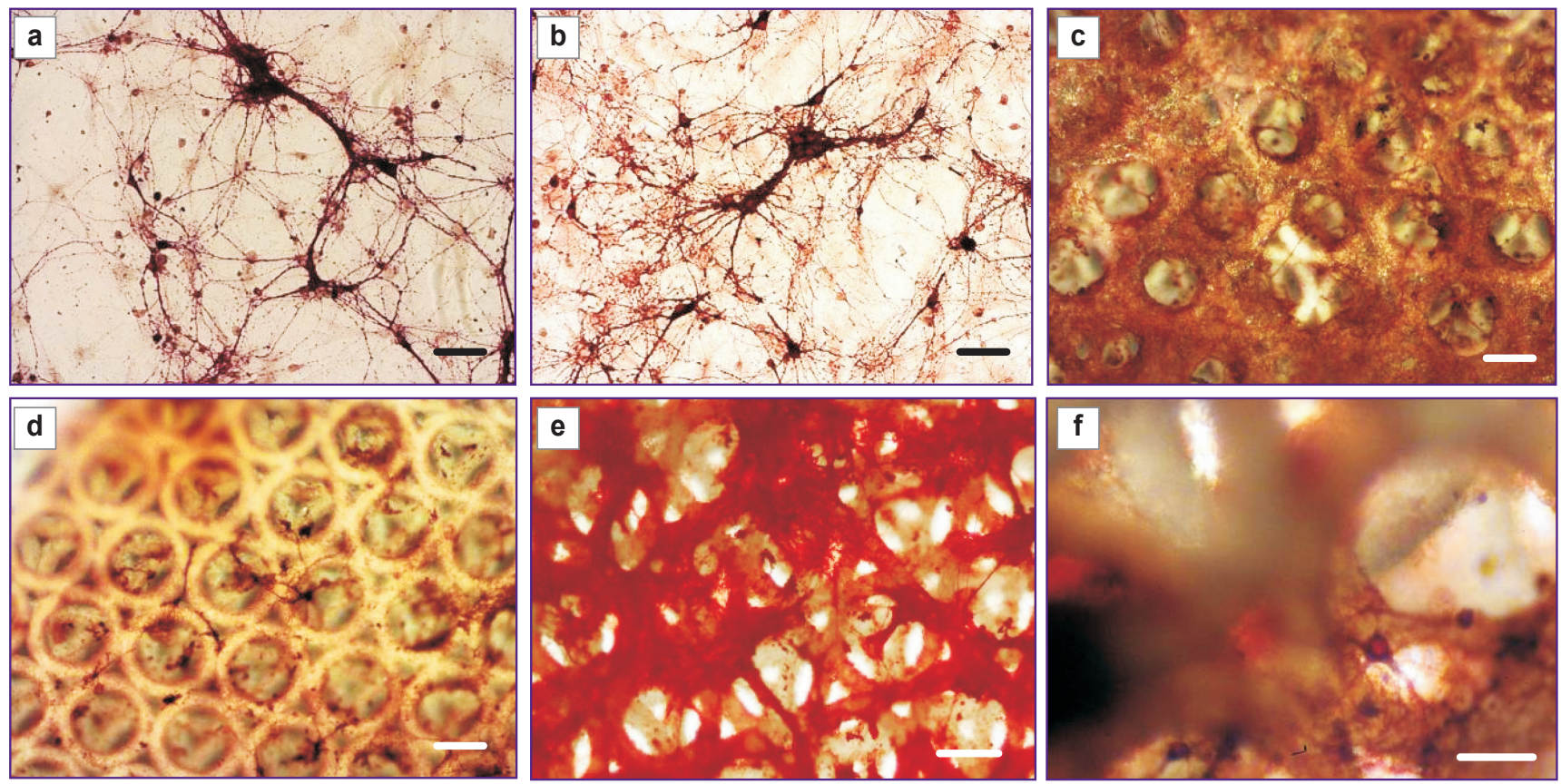

Figure 2. Optic micrographs of immunohistochemical analysis of the dissociated hippocampal culture with neuronal and presynaptic markers on day 10 of the development on the coverslip and scaffold: neuronal marker $\beta$ III-tubulin (a), (c), (e); presynaptic marker synaptophysin (b), (d), (f); scale bar $20 \mu \mathrm{m}$ 


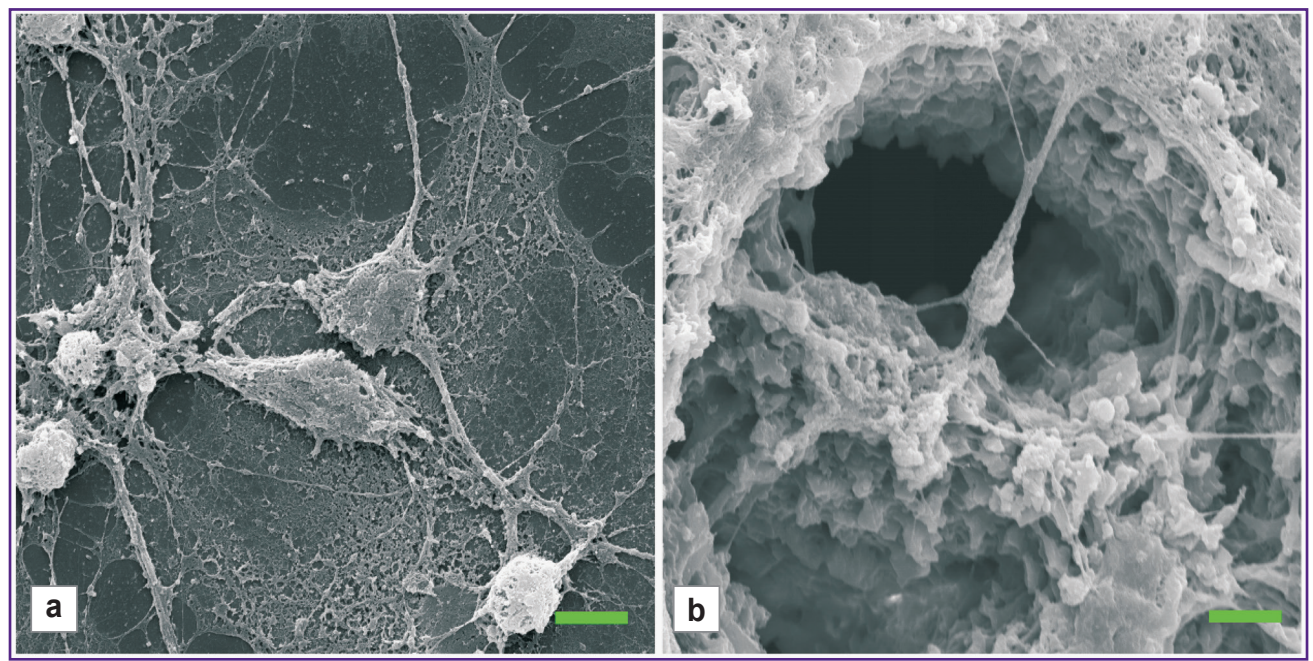

Figure 3. Scanning electron microscopy. Micrographs of the dissociated hippocampal culture on day 12 of the development: control culture on the coverslip (a) and a branched neuronal network formed on the matrix-carrier (b); scale bar $10 \mu \mathrm{m}$

Acknowledgements. The authors thank S.V. Kostyuk for providing the polymer samples, A.A. Frolova for preparing the samples for atomic-force microscopy.

Study Funding. The work was financially supported by the Russian Scientific Fund (Project No.1513-00140, cellular culture of dissociated neurons, immunohistochemical analysis of immobilized culture, SEM neuron culture analysis), and the Russian Foundation for Basic Research (Project No.15-29-04908, $3 \mathrm{D}$ structures forming as a neurotransplantation matrixes, SEM and AFM matrixes analysis).

Conflicts of Interest. The authors declare no conflicts of interest.

\section{References}

1. Huling J., Ko I.K., Atala A., Yoo J.J. Fabrication of biomimetic vascular scaffolds for $3 \mathrm{D}$ tissue constructs using vascular corrosion casts. Acta Biomater 2016; 32: 190-197, https://doi.org/10.1016/j.actbio.2016.01.005.

2. Kuznetsova D.S., Timashev P.S., Bagratashvili V.N., Zagaynova E.V. Scaffold- and cell system-based bone grafts in tissue engineering (review). Sovremennye tehnologii $v$ medicine 2014; 6(4): 201-212.

3. Kuznetsova D.S., Timashev P.S., Dudenkova V.V., Meleshina A.V., Antonov E.A., Krotova L.I., Popov V.K., Bagratashvili V.N., Zagaynova E.V. Comparative analysis of proliferation and viability of multipotent mesenchymal stromal cells in 3D scaffolds with different architectonics. Bull Exp Biol Med 2016; 160(4): 535-541, https://doi.org/10.1007/s10517016-3214-8.

4. Tripathi G., Basu B. A porous hydroxyapatite scaffold for bone tissue engineering: physico-mechanical and biological evaluations. Ceramics International 2012; 38(1): 341-349.

5. Gittard S.D., Nguyen A., Obata K., Koroleva A., Narayan R.J., Chichkov B.N. Fabrication of microscale medical devices by two-photon polymerization with multiple foci via a spatial light modulator. Biomed Opt Express 2011; 2(11): 3167-3178, https://doi.org/10.1364/BOE.2.003167.

6. Ovsianikov A., Malinauskas M., Schlie S., Chichkov B., Gittard S., Narayan R., Löbler M., Sternberg K., Schmitz K.-P., Haverich A. Three-dimensional laser micro- and nano-structuring of acrylated poly(ethylene glycol) materials and evaluation of their cytoxicity for tissue engineering applications. Acta Biomater 2011; 7(3): 967-974, https://doi. org/10.1016/j.actbio.2010.10.023.

7. Gittard S.D., Koroleva A., Nguyen A., Fadeeva E., Gaidukeviciute A., Schlie S., Narayan R.J., Chichkov B. Two-photon polymerization microstructuring in regenerative medicine. Front Biosci (Elite Ed) 2013; 5: 602-609, https://doi. org/10.2741/E642.

8. Sabir M.I., Xu X., Li L. A review on biodegradable polymeric materials for bone tissueengineering applications. J Mater Sci 2009; 44(21): 5713-5724, https://doi.org/10.1007/ s10853-009-3770-7.

9. Timashev P., Kuznetsova D., Koroleva A., Prodanets N., Deiwick A., Piskun Y., Bardakova K., Dzhoyashvili N., Kostjuk S., Zagaynova E., Rochev Y., Chichkov B., Bagratashvili V. Novel biodegradable star-shaped polylactide scaffolds for bone regeneration fabricated by two-photon polymerization. Nanomedicine 2016; 11(9): 1041-1053, https:// doi.org/10.2217/nnm-2015-0022.

10. Biran R., Martin D.C., Tresco P.A. Neuronal cell loss accompanies the brain tissue response to chronically implanted silicon microelectrode arrays. Exp Neurol 2005; 195(1): 115126, https://doi.org/10.1016/j.expneurol.2005.04.020.

11. Williams J.C., Hippensteel J.A., Dilgen J., Shain W., Kipke D.R. Complex impedance spectroscopy for monitoring tissue responses to inserted neural implants. J Neural Eng 2007; 4(4): 410-423, https://doi.org/10.1088/1741-2560/4/4/007.

12. Timashev P.S., Vedunova M.V., Guseva D., Ponimaskin E., Deiwick A., Mishchenko T.A., Mitroshina E.V., Koroleva A.V., Pimashkin A.S., Mukhina I.V., Panchenko V.Ya., Chichkov B.N., Bagratashvili V.N. 3D in vitro platform produced by two-photon polymerization for the analysis of neural network 
formation and function. Biomed Phys Eng Express 2016; 2(3), https://doi.org/10.1088/2057-1976/2/3/035001.

13. Timashev P.S., Bardakova K.N., Minaev N.V., Demina T.S., Mishchenko T.A., Mitroshina E.V., Akovantseva A.A., Koroleva A.V., Asyutin D.S., Pimenova L.F., Konovalov N.A., Akopova T.A., Solov'eva A.B., Mukhina I.V., Vedunova M.V., Chichkov B.N., Bagratashvili V.N. Compatibility of cells of the nervous system with structured biodegradable chitosan-based hydrogel matrices. Applied Biochemistry and Microbiology 2016; 52(5): 508-514, https://doi.org/10.1134/ s0003683816050161.

14. Kobe F., Guseva D., Jensen T.P., Wirth A., Renner U.,
Hess D., Müller M., Medrihan L., Zhang W., Zhang M., Braun K., Westerholz S., Herzog A., Radyushkin K., El-Kordi A., Ehrenreich H., Richter D.W., Rusakov D.A., Ponimaskin E. 5-HT7R/G12 signaling regulates neuronal morphology and function in an age-dependent manner. J Neurosci 2012; 32(9): 2915-2930, https://doi.org/10.1523/ JNEUROSCI.2765-11.2012.

15. Theiler S., Diamantouros S.E., Jockenhoevel S., Keul H., Moeller M. Synthesis and characterization of biodegradable polyester/polyether resins via Michael-type addition. Polymer Chemistry 2011; 2(10): 2273, https://doi. org/10.1039/c1py00262g. 